\title{
Research and Application of Power Transmission Line On-line Monitoring Device in Alpine Regions
}

\author{
Jian Zhang ${ }^{1, a}$, Xuecheng Zhu ${ }^{1, b}$, Ziwei Gao ${ }^{1, c}$, Wei Sun ${ }^{1, d}$, Yang Zhou ${ }^{1, \text { e }}$, \\ Hongyi Zhou ${ }^{1, f}$ and Haiming Sun ${ }^{1, g}$ \\ ${ }^{1}$ Electric Power Research Institute of State Grid Heilongjiang Electric Power Company Limited, \\ Harbin, China \\ a13946077726@139.com, ${ }^{b}$ zhuxuecheng2004@163.com, ${ }^{c}$ gaoziwei1979@163.com, \\ d sunwei19850525@163.com, ${ }^{\mathrm{e}}$ 124419058@qq.com, ${ }^{\mathrm{r}}$ 84219292@qq.com, ${ }^{\mathrm{g}}$ epushmbj@126.com
}

Keywords: On-line Monitoring, Alpine Region, Power Transmission Line, Aeolian Vibration. Abstract. China is characterized by vast territory and wider distribution of strong wind easy-happening areas (typhoon, tornado and squall line wind). Meteorological environment conditions are complex and harsh in power transmission line corridor passage (especially in alpine regions). Safe operation of power transmission lines is threatened severely by strong wind natural disaster. Traditional technology can't meet the requirement of power grid rapid development on anti-disaster ability. Large-area tower collapse, cable breakage and other catastrophic wind disaster caused by strong wind in alpine regions as well as control techniques thereof are not studied deeply. The applicability of power transmission line on-line monitoring devices in alpine regions still should be further evaluated. In the paper, long-span guide wire aeolian vibration safety in alpine regions and evaluation methods thereof as well as the wind-induced fatigue early-warning model of long-span guide wires were studied. Long-span guide wire aeolian vibration evaluation standard in alpine regions and fatigue damage early-warning model based on rotatory motion effect index were proposed. The blank in the research field of alpine region long-span guide wire aeolian vibration was remedied. In addition, the guide wire wind resistance safety monitoring project of long-span lines in alpine regions was applied. Continuous valid data for more than one year were obtained, thereby providing basis for formulating guide wire aeolian vibration vibration control measures.

\section{Introduction}

Strong wind disasters cause tower collapse, cable breakage and tripping accidents of power transmission and distribution lines frequently. Large-area power outage can be produced sometimes with extremely high uncertainty and abiogeny, thereby leading to great difficulty on wind disaster prevention and treatment in power transmission and distribution lines. In addition, Alpine regions account for $27.9 \%$ or so of total land area in China. Energy-reach Northeast China, Inner Mongolia, Northwest China and other regions mostly belong to alpine regions. In addition, the minimum temperature was $-59{ }^{\circ} \mathrm{C}$ in the arctic with the implementation of the global energy Internet strategy. Improvement of cryogenic reliability in power transmission lines becomes a major technical problem that should be solved ${ }^{[1]}$.

On-line monitoring devices provide power transmission equipment with functions of displaying, early-warning, analyzing, diagnosing, evaluating and predicting state information. State monitoring data are provided for other related systems intensively, thereby realizing comprehensive monitoring of power transmission equipment state and state operation management ${ }^{[2]}$. In recent years, State Grid Corporation of China engaged in certain research on prevention and management of strong wind disasters. However, large-area tower collapse, cable breakage and other catastrophic wind disaster caused by strong wind in alpine regions as well as control techniques thereof were not studied deeply. The applicability of power transmission line on-line monitoring devices in alpine regions still should be further evaluated ${ }^{[3]}$. In the paper, long-span guide wire aeolian vibration safety in alpine regions and evaluation methods thereof as well as the wind-induced fatigue early-warning model of long-span guide wires were studied. In addition, the guide wire wind resistance safety monitoring project of 
long-span lines in alpine regions was applied. The blank in the research field of alpine region long-span guide wire aeolian vibration was remedied.

\section{Research on power transmission line aeolian vibration on-line monitoring device in alpine regions}

Research on wire aeolian vibration. Aeolian vibration undergoes on-line monitoring. Long-term continuous measurement is realized for obtaining accurate measurement data, analyzing and judging aeolian vibration level and wire fatigue life on the one hand. Failures can be diagnosed conveniently, reasons can be analyzed and discovered on the other hand, thereby facilitating research on the relationship between guide wire vibration level and meteorology conditions, discovering hidden danger of guide wire vibration as early as possible, eliminating failures timely, and improving the safety and reliability of line operation greatly. Measurement of field aeolian vibration has the following functions ${ }^{[4]}$ :

a) Measure guide wire vibration response in the field, understand actual vibration level of guide wires, judge whether vibration is qualified or not, quantitatively analyze and assess vibration risks, and evaluate cable breakage risk degree;

b) Quickly detect the location in the guide wire with vibration hidden danger;

c) Evaluate the effect of the vibration control plan on the line, and provide basis for correctly selecting and positing vibration control elements; recognize and test the vibration control plan necessary before handover of the newly-built lines;

d) Evaluate the vibration strength of damaged guide wires, confirm whether the wire is damaged by vibration or not, and adopt preventive measures to prevent further damage;

e) Establish reasonable inspection time interval and establish an maintenance plan;

f) Evaluate vibration safety of the line (especially old lines);

g) Verify the theory of vibration.

Long-span aeolian vibration on-line monitoring device in alpine region. Aeolian vibration of overhead power transmission line guide wires is the main reason of guide wire fatigue damage. Guide wire dynamic bending strain is a main parameter to express the fatigue damage degree. However, it is not practical to measure the dynamic bending strain of wires directly under field operation conditions except special study. Bending amplitude method was presented by IEEE on the basis of study and practice for many years.

Bending amplitude is based on relative amplitude of two points. One point is the terminal contact point $\mathrm{O}$ of the wire guide and wire clamp. The other point is point $\mathrm{b} 89 \mathrm{~m}$ away from point $\mathrm{O}$. The relative lateral amplitude $Y_{b}(P-P)$ of point $b$ and point $O$ is the bending amplitude as shown in Fig.1. There is an estimated and basically linear relationship between bending amplitude value and dynamic bending strain. The relationship is not related to the connection method of vibration frequency and the wire clamp. It is proved by practice that bending amplitude is a simple and practical method to measure guide wire vibration in the wire clamp. The vibration in the point $89 \mathrm{~mm}$ away from the wire clamp is measured. Poffemberger-Swart calculation method is applied for calculating the strain of the wire clamp outlet as shown in Eq.1.

$\varepsilon=\frac{E_{\alpha} D p^{2} Y_{b}}{e^{-p x}-I+p x}$

In the formula:

$\mathrm{E}_{\mathrm{a}}-$ Young's modulus of outer strand, MPa;

$\mathrm{D}$ refers to wire diameter, $\mathrm{mm}$;

$\mathrm{x}=89 \mathrm{~mm}$;

$\mathrm{Y}_{\mathrm{b}}$ refers to the peak value of vibration park at $89 \mathrm{~mm}$, $\mathrm{mm}$; 
P refers to experimental constant, which is related to wire tension $\mathrm{T}$ (unit: $\mathrm{N}$ ) and wire flexural rigidity EI (unit: $\mathrm{N} \cdot \mathrm{m})$ :

$$
p=\sqrt{\frac{T}{E I}}
$$

Fig.1 shows that class II network structures are adopted for the aeolian vibration monitoring system. The structure is composed of vibration monitoring unit, meteorology monitoring unit, on-line monitoring base station and local monitoring center, wherein the vibration monitoring unit is installed on the guide wire, the meteorology monitoring unit and the on-line monitoring base station are installed on the tower, and the local monitoring center is set on the rear end.

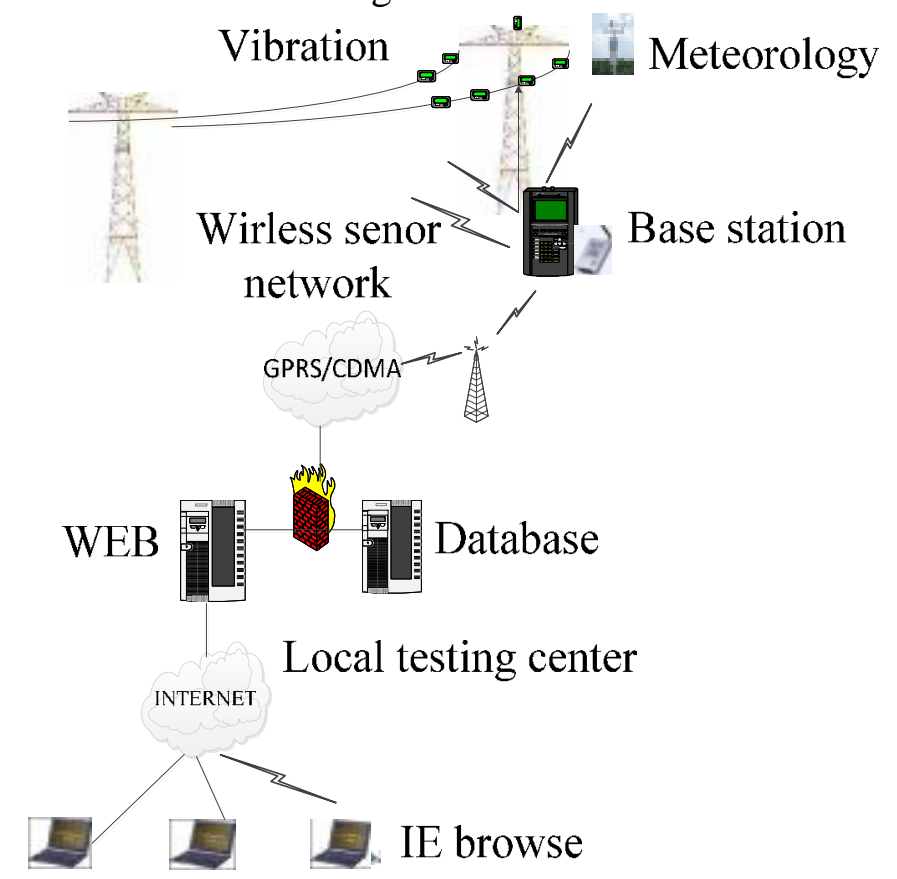

Figure 1 Network diagram of aeolian vibration monitoring system.

Aeolian vibration monitoring devices are installed in guide wires and OPGW fatigue risk points according to indoor test results and field operation experience of vibration control devices. Typical positions generally include damper line chucks, damper chuck, spacer chuck, suspension clamp outlet, armor road terminal, tension joint splice terminal, etc as shown in Fig.2 and Fig.3.

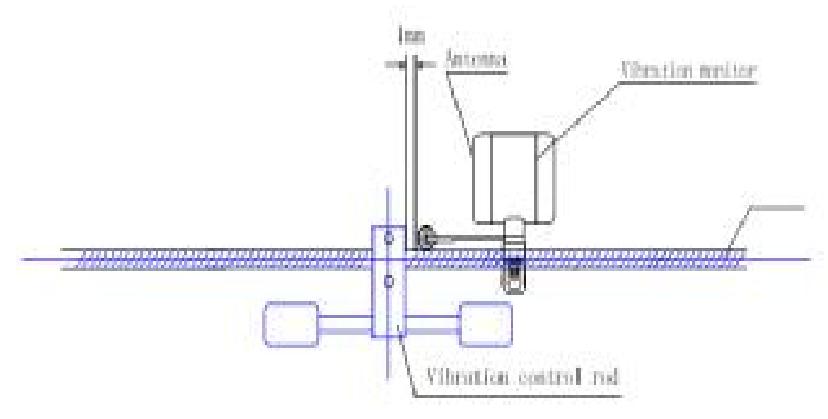

Figure 2 Schematic diagram of wire vibration collection unit installation.

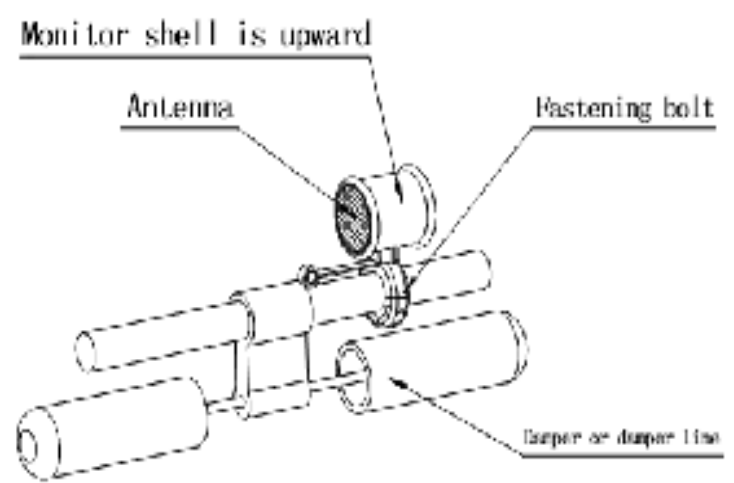

Figure 3 Schematic diagram of ground wire and OPGW vibration collection unit installation.

\section{Application of power transmission line on-line monitoring device in alpine region}

Aeolian vibration on-line monitoring project in Hemu Line B. In the paper, Heilongjiang Hemu Line B Project was selected for installation and data monitoring of aeolian vibration monitoring 
systems. The condition requirements in the alpine region were satisfied. Installation environment in Hemu Line B was shown in Fig.4. Aeolian vibration monitor was shown in Fig.5 and Fig.6. Aeolian vibration monitoring base station was shown in Fig.7.

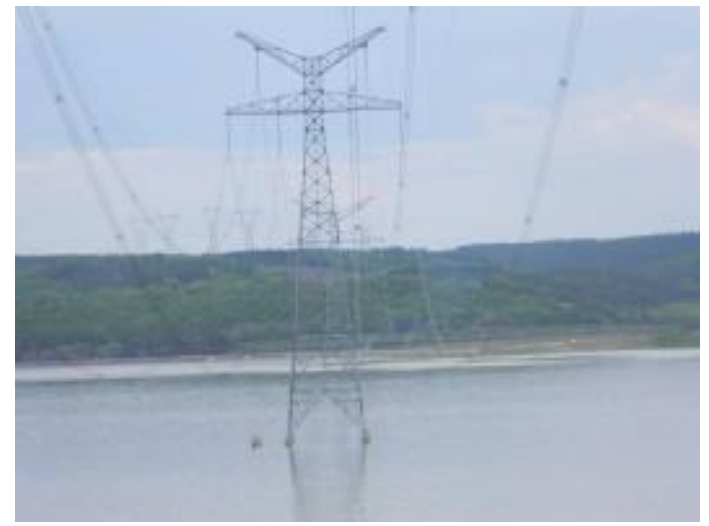

Figure 4 Installation environment of aeolian vibration on-line monitoring system in Hemu Line B.

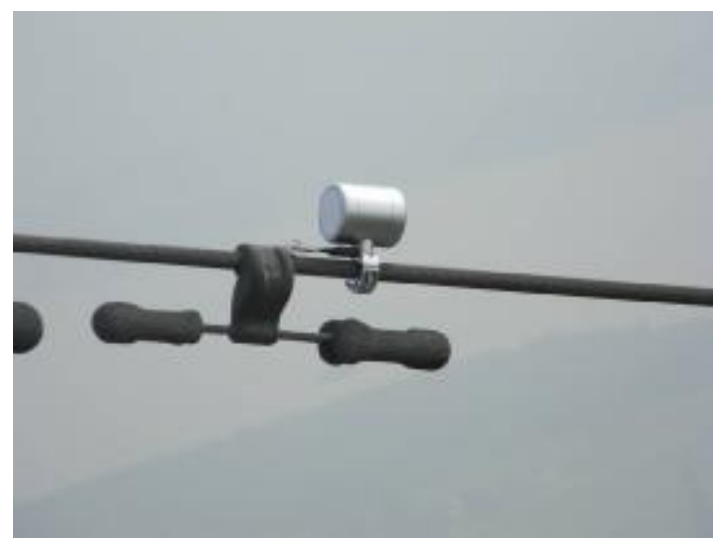

Figure 6 Aeolian vibration monitor.

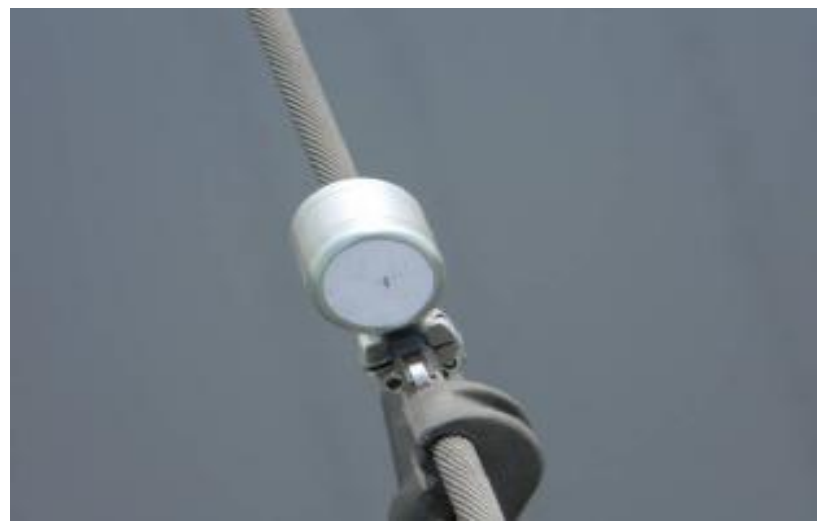

Figure 5 Aeolian vibration monitor.

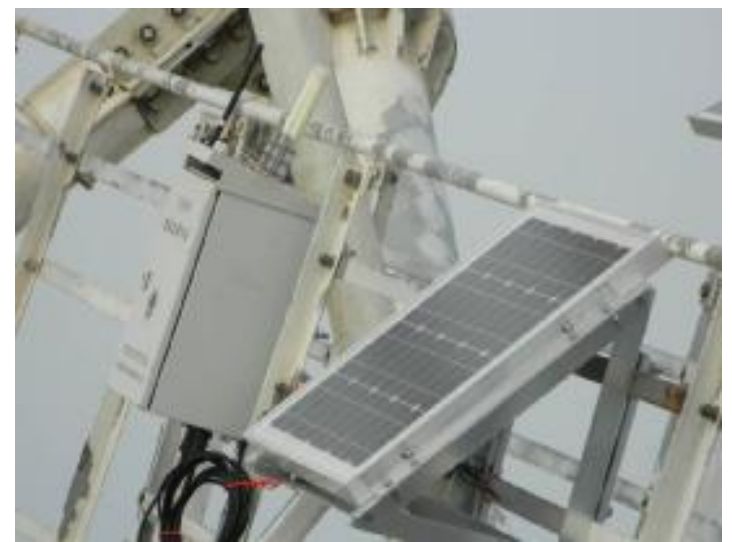

Figure 7 Aeolian vibration monitoring base station.

Environmental data collection in Hemu Line B. Minimum environment temperature was $-27.6^{\circ} \mathrm{C}$, and the measurement time was respectively 2013-01-09 07:40 (wind speed: $6.6 \mathrm{~m} / \mathrm{s}$ ) 7:50 $(7.5 \mathrm{~m} / \mathrm{s}$ ) and 2012-12-26 04:10:05 (wind speed: $9.8 \mathrm{~m} / \mathrm{s})$. Maximum temperature was $33.9^{\circ} \mathrm{C}$, and measurement time was 2013-06-25 14:20:05 (wind speed: $0.4 \mathrm{~m} / \mathrm{s}$ ).

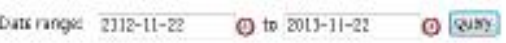

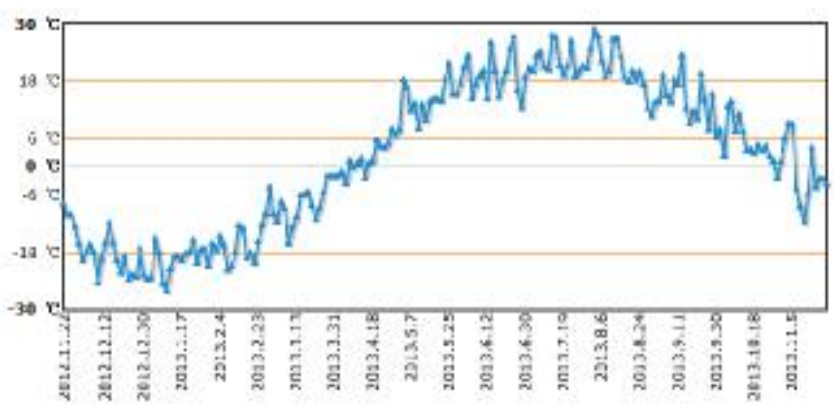

Figure 8 Atmospheric temperature data on the tower (whole-year from 2012.11.22 to 2013.11.22).

Maximum wind speed was $23.1 \mathrm{~m} / \mathrm{s}$, and the measurement time was 2013-11-18 03:20:06 (atmospheric temperature: $-3.3^{\circ} \mathrm{C}$ ). 


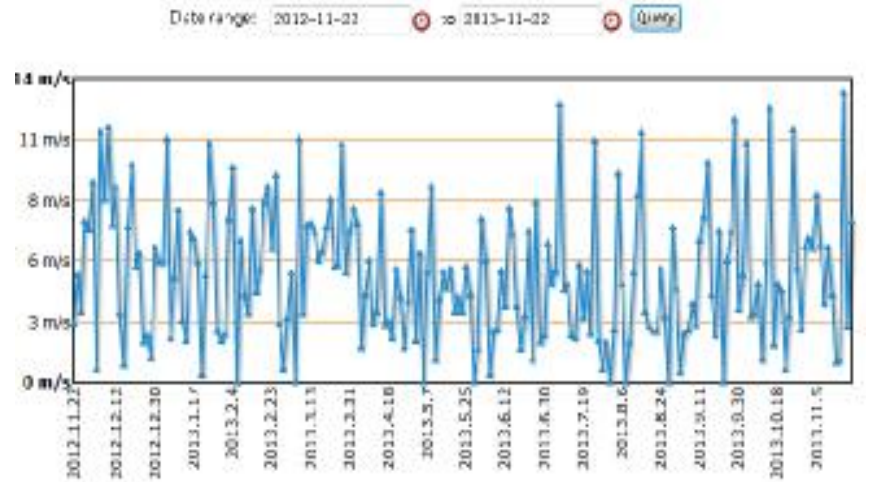

Figure 9 Wind speed data.

(whole-year from 2012.11.22 to 2013.11.22)

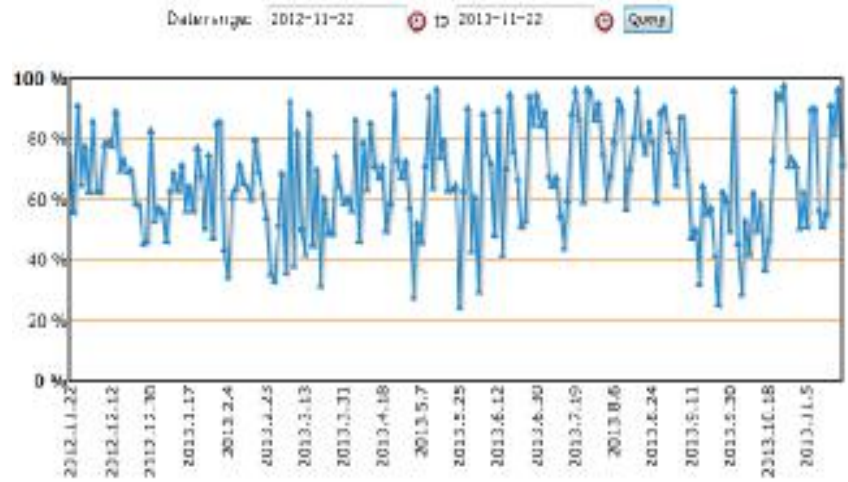

Figure 10 Humidity data. (whole-year from 2012.11.22 to 2013.11.22)

Wire temperature data of No. 101 tower in Hemu Line B. The section shows changes of wire temperature within one year. The aeolian vibration monitor is equipped with two wire temperature sensors, which are installed around the monitor.

Wire temperature measurement data of bottom-phase wire 1\# measurement (No. 552 monitor) was listed in Fig.11. Measurement results showed that maximum wire temperature was $40.4^{\circ} \mathrm{C}$, and the measurement time was respectively 2013-07-17 14:12:51 and 2013-06-25 12:33:11.

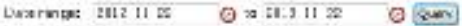

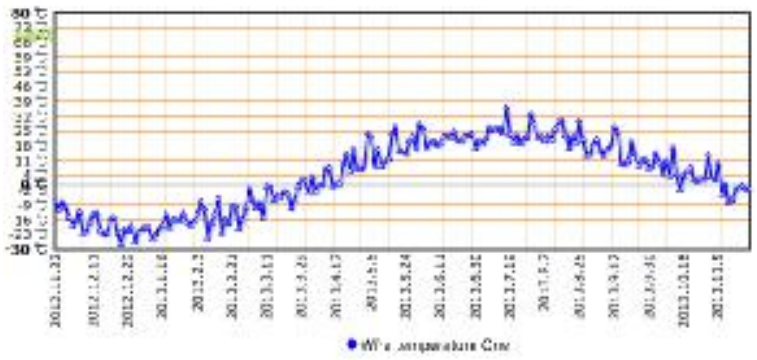

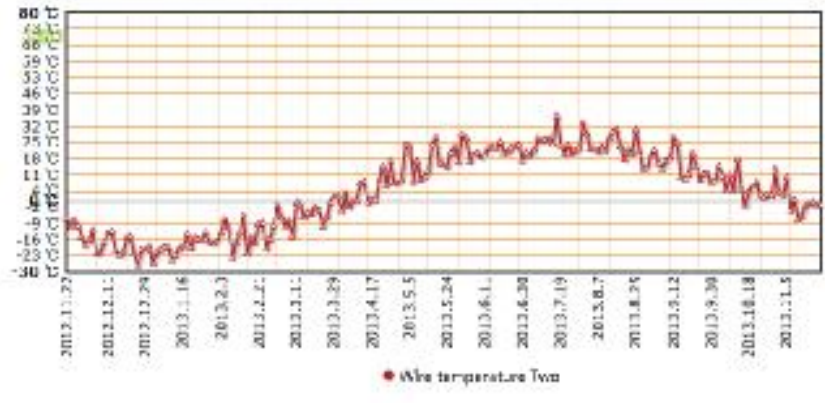

- Whe tareneratre Twre

Figure 11 Wire temperature data of No. 552 monitor (2012.11.22-2013.11.22).

Wire temperature measurement data of bottom-phase wire 2\# temperature measurement point (No. 553 monitor) was listed in Fig. 12 . The maximum temperature was $40.7^{\circ} \mathrm{C}$, and the measurement time was 2013-07-17 13:52:59.

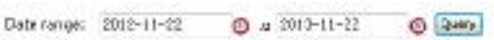

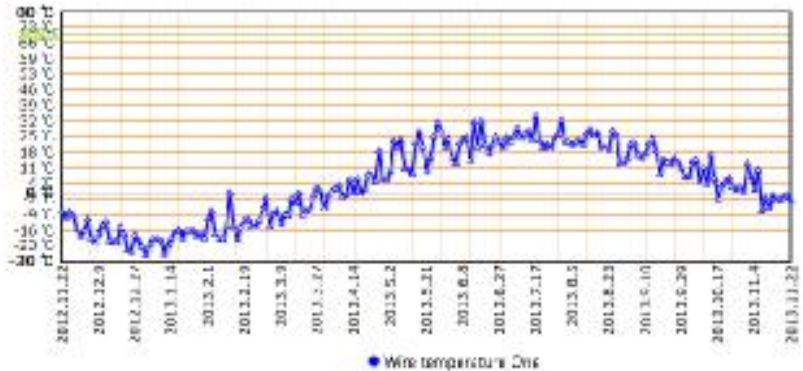

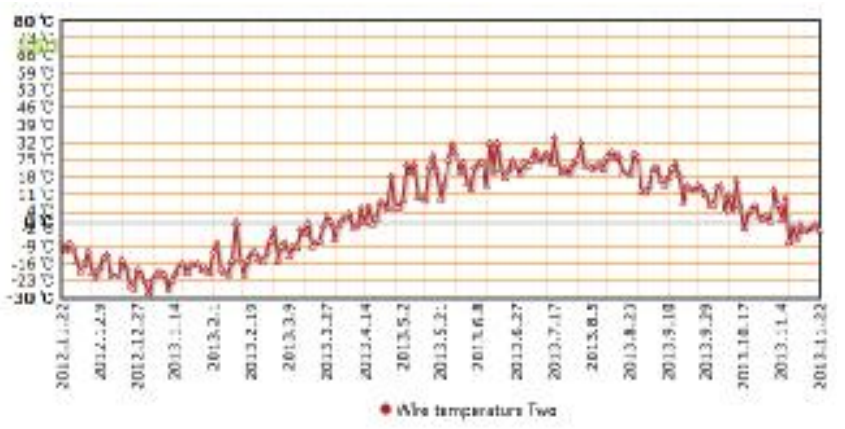

Figure 12 Wire temperature data of No. 553 monitor (2012.11.22-2013.11.22).

Wire temperature measurement data of bottom-phase wire 3\# measurement (No. 554 monitor was shown in Fig.13. Wire the maximum temperature was $41.1^{\circ} \mathrm{C}$, and the measurement time was 2013-06-25 12:03:04. 

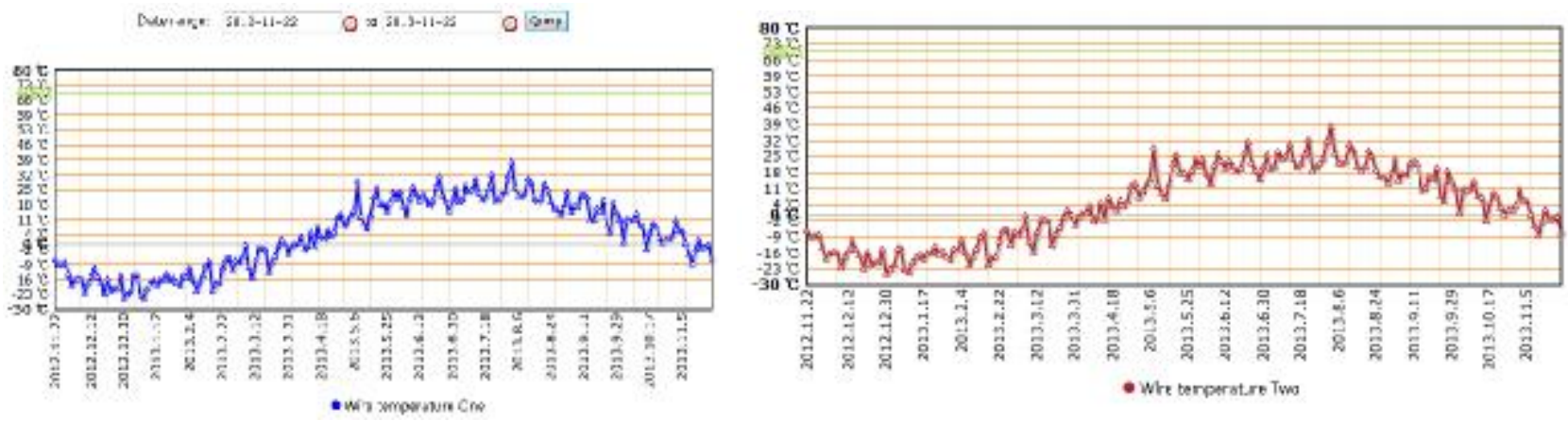

Figure 13 Wire temperature data of No. 554 monitor (2012.11.22-2013.11.22).

Aeolian vibration data of No. 101 tower in Hemu Line B. Vibration measurement data of No. 552 monitor during monitoring was listed in Fig. 14. Measurement results showed that the maximum dynamic bending strain value was the result at 11:08:24 on 2012-11-22 $\pm 33.620 \mu \varepsilon$ when aeolian vibration was within the range of $\pm 1 \sim \pm 50 \mu \varepsilon$, which was lower than the allowable value of wire dynamic bending strain.

Vibration measurement data of No. 553 monitor during monitoring was listed in Fig.15. Measurement results showed that the maximum dynamic bending strain value was the result at 16:09:41 on 2012-11-24 $\pm 30.560 \mu \varepsilon$ when aeolian vibration was within the range of $\pm 1 \sim \pm 50 \mu \varepsilon$ which was lower than the allowable value of wire dynamic bending strain.

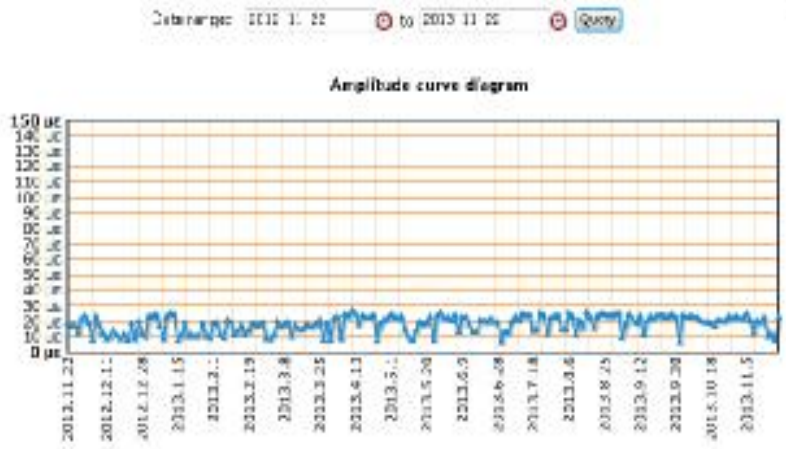

Figure 14 Amplitude curve data of No. 552 monitor(2012.11.22-2013.11.22).

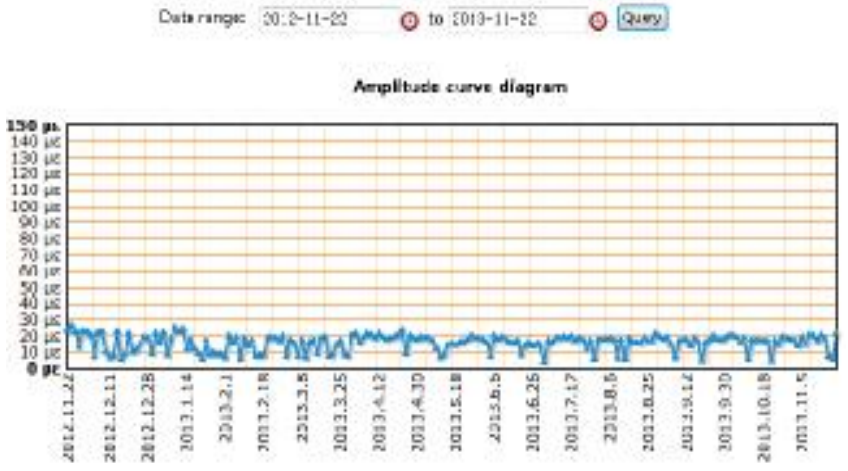

Figure 15 Amplitude curve data of No. 553 monitor (2012.11.22-2013.11.22).

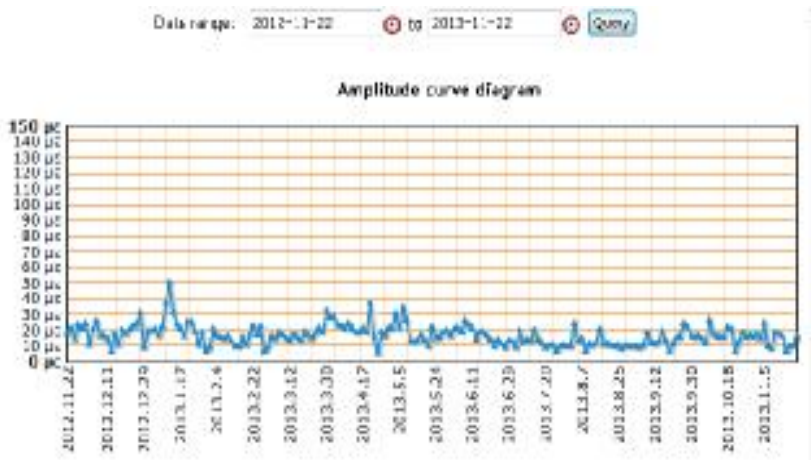

Figure 16 Amplitude curve data of No. 554 monitor (2012.11.22-2013.11.22).

Vibration measurement data of No. 554 monitor during monitoring was listed in Fig.16. Measurement results showed that the maximum dynamic bending strain value was the result at 17:39:47 on 2012-11-24 $\pm 101.620 \mu \varepsilon$ when aeolian vibration was within the range of $\pm 1 \sim \pm 102 \mu \varepsilon$, which was lower than the allowable value of wire dynamic bending strain. 


\section{Conclusion}

In the paper, long-span guide wire aeolian vibration safety in alpine regions and evaluation methods thereof as well as the wind-induced fatigue early-warning model of long-span guide wires were studied. Long-span guide wire aeolian vibration evaluation standard in alpine regions and fatigue damage early-warning model based on rotatory motion effect index were proposed. The blank in the research field of alpine region long-span guide wire aeolian vibration was remedied. In addition, the guide wire wind resistance safety monitoring project of long-span lines in alpine regions was applied. Continuous valid data for more than one year were obtained. Vibration was lowered to a safer scope through increasing and adjusting damper and other measure, thereby providing basis for formulating guide wire aeolian vibration vibration control measures.

There were extremely few research results on low temperature in engineering application at home and abroad because of the limitation of experimental conditions. The actual measurement was realized under more different conditions with continuous development of on-line monitoring technology, thereby driving in-depth research on low temperature performance of power transmission line towers, wires, hardware and other parts. More detailed and concrete anti-wind measures were established aiming at anti-wind damage in the alpine region, and systematic anti-wind equipment was developed on the basis of study. Meanwhile, the research on anti-wind theory in the alpine region will be driven by development of multi-dimensional measurement, 'large data' and other techniques, thereby further improving the development of fault intelligent identification and warning function.

\section{References}

[1] Sun Caixin. Current status and prospect of state on-line monitoring and diagnostic technology of power transmission and conversion equipment. China Power, 2005, 38 (2):1-7

[2] Zhang Yiqun, Li Haixing, Analysis and prevention of typical failure cases of power transmission line. China Power Press, 2012

[3] Zou Jianming. Application of on-line monitoring technology in the power grid. High Piezoelectric Technology, 2007, 203-206

[4] Han Xiaorong. Zhong Yeqian. Analysis on current status and prospect of state on-line monitoring and diagnostic technology of power transmission and conversion equipment. Super Technology, 2013 (12), 94-95 